\title{
Application of Space Consciousness in Craft Carving - Taking Huian Stone Carving as an Example
}

\author{
Lin Zibo ${ }^{1, \mathrm{a}}, \mathrm{Hu} \mathrm{Jiawei}^{2, \mathrm{~b}^{*}}$ \\ ${ }^{1}$ Xiamen Academy of Arts and Design, Fuzhou University, Xiamen, Fujian, China \\ ${ }^{2}$ Xiamen Academy of Arts and Design, Fuzhou University, Xiamen, Fujian, China \\ a57762087@qq.com \\ b*122760844@qq.com
}

\begin{abstract}
Craft carving is an art with a long history. From carving hunting tools in the stone age to daily utensils in Yangshao culture, to exquisite decorations in the Ming and Qing Dynasties, craft carving is closely related to the daily life of the broad masses of the people; Huian, China, is the capital of stone carving in the world. Its 1600 years of carving inheritance plays an important role in contemporary stone carving; Craft carving and sculpture are interlinked, but different from sculpture. They are all studying the art of three-dimensional space. Space is composed of positive space, that is, noumenon space and negative space. This paper will connect negative space consciousness with craft carving, so as to broaden the space consciousness of craft carving to a great extent and make its works more possible and accidental.
\end{abstract}

Keywords: craft carving, Huian, space

\section{工艺雕刻中空间意识的应用一以惠安石雕为例}

\author{
林梓波 ${ }^{1, \mathrm{a}}$,胡嘉炜 $2, \mathrm{~b}^{*}$
}

${ }^{1}$ 福州大学厦门工艺美术学院, 厦门, 福建, 中国

2 福州大学厦门工艺美术学院, 厦门, 福建, 中国

a57762087@qq.com

b*122760844@qq.com

\section{摘要:}

工艺雕刻是一门历史悠久的艺术，从石器时代雕刻狩猎工具到仰韶文化的日用器具再到明清的精致装饰品，一 直到现在工艺雕刻品都与广大人民群众的日常生活密不可分; 中国惠安是世界石雕之都，其 1600 年来的工艺 雕刻传承在当代工艺石雕中有着举足轻重的影响; 工艺雕刻与雕塑相通却又区别于雕塑, 其根本都是在研究立 体空间的艺术，空间是由正空间即本体空间和负空间所组成的，本文将把负空间意识与工艺雕刻相连接，从很 大程度上去扩宽工艺雕刻品的空间意识, 让其作品有了更多的可能性和偶然性。

关键词: 工艺雕刻、惠安、空间

\section{1. 前言}

中国工艺雕刻最早可以追溯到原始社会时期, 是 通过对实用工具不断的探索研究发展而来的; 到了战 国初期, 其工艺雕刻也已经初具规模, 汉代的动物雕 刻也将其工艺雕刻艺术推向了一个高点; 再到唐宋时 期, 随着文化的大繁荣和外来文化的融入交流, 其工 艺雕刻门类已日趋完善; 到元明则是古代工艺雕刻的
最高点; 对于近代而言, 随着手工业不断的发展, 加 之工业化的进步和世界极其不稳定的关系使得整个 工艺雕刻行业经历了起起落落; 进入二十一世纪以 来, 世界走向 “地球村” , 我国受改革开放的影响经 济大繁荣, 国家也大力提倡手工业复兴, 加强非物质 文化遗产的保护, 使得工艺雕刻发展再次迈入新的台 阶; 加以 3D 打印技术的日趋完善, 也为其工艺雕刻 行业的革新提供了更多的思路。 


\section{2. 惠安工艺雕刻概述}

惠安石雕是中国福建省的传统雕刻技艺之一, 作 为中国优秀传统文化的一朵奇菂, 历经一千多年的繁 衍发展, 仍然保留着非常纯粹的中国艺术传统, 保持 着很完整的延续性, 至今未被西方外来文化所异化, 具有强烈的民族性。

早在 1600 多年前的晋朝, 惠安石雕作为永久性 的可存性艺术已被应用。建国后, 惠安石雕工艺精益 求精、日臻完善。惠安石雕早期主要服务于宗教, 具 有浓厚的宗教色彩。主要体现在宫观寺庙的建筑设 计、雕刻安装, 寺内外塔、亭、柱、栏等的建造雕刻, 以及对神佛造像出神入化的雕刻上。现代惠安石雕创 作手法十分复杂，有着多种表现方法，圆、浮、透、 线、沉、影等多种雕法并存, 大者雄伟壮观，小者精 微细巧, 创作题材广泛, 人物、动物、故事、场景, 或情景交融，或针砭时弊，体现了 “自然与理性结合 之美”。除了单纯的艺术享受, 作品还体现出了人文 关怀和对社会现象的思考。

\section{3. 空间的阐述}

工艺雕刻是在展现其精湛工艺的同时注重作品 本身空间的艺术, 在现当代工艺雕刻作品中, 随着工 艺技术的完善使得工艺雕刻师、设计师都把创新的重 点放在了其作品形体和空间的关系上, 亨利 - 摩尔 说: “我发现形体和空间其实是同一回事。”可见若 没有把握其工艺雕刻作品的形体, 也就没有抓住其作 品的空间, 反之也成立即不能把握空间就不能把握好 作品的形体, 就像宇宙就是一个空间, 这个空间的形 成和组建是通过一个小小的点即地球而被感知出来 的, 通过对宇宙空间的构建让人产生了对地球以外的 无限遐想, 这是一个极其抽象的概念。放到工艺雕刻 作品中一样适用, 优秀的工艺雕刻作品都是通过精美 的作品让观者产生无限的遐想空间, 这种空间不是二 维的平面空间也不是三维的立体空间，而是类似于四 维或更高的抽象空间, 如果没有工艺雕刻品的存在那 么也就没有这种空间存在的条件, 所以负空间 (抽象) 是相对于正空间（立体空间）而言的，本文将对现代 工艺雕刻作品的空间进行讨论, 让观赏者在作品给予 的空间中自我探寻或思考一些容易被忽视的、潜意识 的、被日常审美所阻碍的非物质之美。

在现实生活中的空间是可以让人感知的, 是具体 存在的、可以触摸到的, 就像自己的房间或是公共的 操场等等; 而能被感知到的另一种摸不到看不着抽 象、虚无、多变的就是负空间, 它是被人的知觉感知 而产生的, 并且随着人认知的改变而改变, 它是人与 物之间相互作用而产生的认知结果, 就像一张白纸放 在桌面上它只是一个平面没有空间感, 但人为的给纸 张折痕或是揉搓使之形变它就会产生一个简单的空 间，而这个简单的空间不是纸张本身所具有的而是人 们感知所给予的; 同样, 复杂的负空间也是由无数这 样无限循环的简单空间而构成的, 因此对负空间的感
知就一定要通过物体; 恰好这样的原理适用于艺术作 品之中, 尤其是三维立体的工艺雕刻作品, 通过观赏 者与作品之间的相互影响而产生因人而异内容丰富 的空间。

\section{4. 工艺雕刻作品的空间体现}

工艺雕刻本身就是将石材、木材等材料通过人为 的艺术处理使之变成工艺美术作品, 丰富人们的日常 生活。而雕刻具有正负空间，雕刻作品本身的形体被 称为正空间, 而很多镂空、表现等需要观者思考理解 的空间就是雕刻作品的负空间。其实这种负空间的概 念早在我国禅文化中的圆融所体现，在宋、明时期的 雕刻作品中出现了大量的 “留白” 作品, 而这种留白 的思考过程就是透过作品本身的空间去寻找负空间。 我国著名艺术家朱尚喜说: “把塑造的虚空间（负空 间）假设成形的塑造，虚空间也应该是有形的。”， 他肯定了其负空间在艺术作品、雕刻作品中应用的意 义。

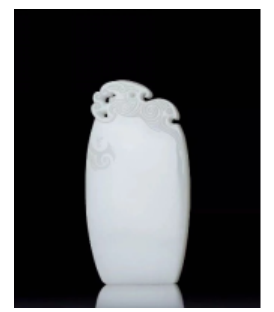

图 1 汉白玉无事牌作品

上文中所提到的留白设计, 这样的处理效果是雕 刻师精心构思设计的, 并不是因为雕艺不精而留下石 材原本的颜色、质地，这就是在雕刻中虚空间的表现 手法, 它这样的处理是为了引导人们的审美思维并不 只在其作品本身而是向作品外的负空间进行无限的 延申，使得雕刻作品拥有作品之外更多的思考、想象 空间。如从明朝末年玉雕大师陆子冈所创的子冈牌, 又称无事牌, 整个雕刻作品没有纹样的装饰, 一直流 传至今深受人们的喜爱，“无即是有” 即什么都没有 就代表什么都有。这是禅文化中 “空” 的体现也正是 负空间意识的体现。由上图所示，整个汉白玉无事牌 虽没有过多雕刻，但是处处都给人以无限遐想的空 间，这就是透过其作品本身，通过 “空” 来产生的非 物质的负空间意境，这种意境的产生也是依托于这个 汉白玉本身所带的气场, 汉白玉是玉中之王, 以温润 纯净的玉质深受人们的追崇, 这种对材料的理解也是 通过人对玉的不断认识而不断改变的。（如图 1）

由此可见，其实在还没有负空间这一名词出现时 的宋、明时期，已经出现了零星的极具负空间的工艺 雕刻作品; 着眼于近代, 工艺雕刻作品五花八门, 笔 者深入世界石雕之都惠安进行实地调研, 在此次调研 中发现了极具负空间语言的优秀当代雕刻作品。 


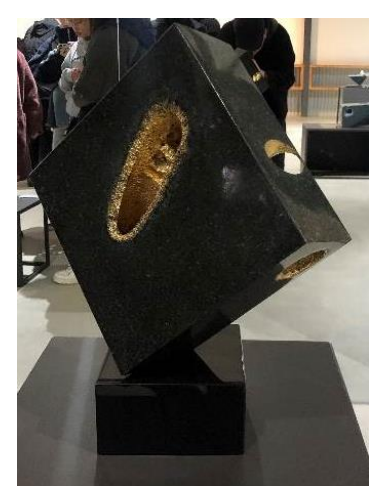

图 2 惠安石雕作品《关系》

《关系》这件工艺雕刻作品就是其负空间意识的 代表; 先从正空间上来分析, 这个应用了石材中较为 稳定的 654 山西黑的材料, 整个作品基本型就是一个 长方体, 而在长方体中艺术家用简单的穿孔机器打通 了 3 到 4 个通道, 有的通道相互连接有的管道却即将 破裂, 并在每个通道上布满了金漆, 作者表达的是其 人身体中血管的一小个角落, 因为人与人、国与国之 间利益就代表着关系所以作品中通道上的金漆就代 表着流动着的血液, 从正空间角度分析发现这个作品 十分的耐人寻味; 但从负空间来分析, 我们透过前文 中对这件设计作品正空间的梳理可以得知这是链接 身体各个器官的血管也就是说作品之外血管 (通道) 的两端就象征着各个脏器, 而各个脏器之间只有通过 血液的流动才能使之正常运作, 这样的负空间就是暗 指现在全球一体化背景下各个国家之间紧密相连的 关系, 同时有的血管是相互连接且十分的笔直畅通这 就暗喻着有些国家之间的关系十分和谐甚至出现的 盟友等情况, 当然在作品正空间中也出现了即将断裂 苃苃可危的血管而这样的血管就暗喻着紧张的国际 形势, 稍有一方比较激进则这个血管也就发生破裂; 当然, 这样关系的理解放在人与人之间是一样行得通 的, 笔者只是从自己的认识方法去解读《关系》这件 作品的其中一个负空间, 正如上文中提到由于每个人 意识形态不同会产生无数个让人沉醉的负空间, 而这 种通过作品而进行无限遐想、沉醉的负空间正是当代 审美下人们所接受所欣赏的。

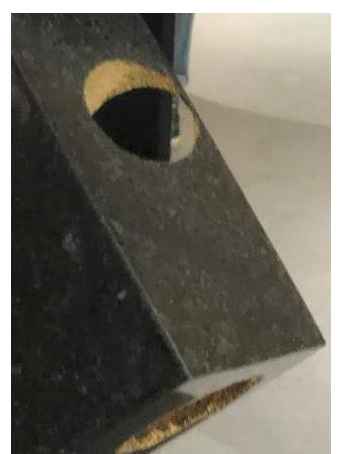

图 3 惠安石雕作品《关系》局部

可见，在当代工艺雕刻品对作品负空间的设计、 研究越来越多, 其作品也由对纹样、材料的研究转向 了对作品正空间影响下负空间的探索; 所以当代雕刻
中空间意识的探讨和研究, 对今后当代工艺雕刻创新 有着一定的积极的作用。

\section{5. 工艺雕刻的负空间形态发展}

工艺雕刻历史悠久是中华民族优秀传统文化, 近 些年来在其发展上遇到了瓶颈; 为改善其创新的问题 笔者将从空间形态中负空间性、心理层面以及意向层 面共三个方向对其在当代雕刻作品上的发展进行阐 述。

工艺雕刻通过上千年来的积累沉淀使之雕刻技 艺精湛, 但由于大多数工艺雕刻技艺都是以材料上的 装饰性浮雕、高浮雕为设计的基础, 使之在三维立体 的正空间感上表现极弱。而这种空间感不强趋于传统 纹样的雕刻作品很难被当代的社会审美所接受; 所以 要通过增加对工艺雕刻作品中三维立体空间的构建 设计, 改善这一现状; 通过对作品正空间的重新架构 使之在正空间上极具视觉冲击力, 同时也能让观赏者 透过作品的正空间去思考作品负空间的意图; 即就像 上文中提到只有保证了正空间的存在才能达到负空 间的建立, 负空间也时时刻刻受到正空间的影响; 所 以, 提高雕刻作品中的三维立体空间一定程度也有利 于当代雕刻作品被更多人所接受。

其次, 就是空间的心理层面; 其心理层面就是以 感知体验为主, 它往往是存在于人们潜意识层面上的 认知。工艺雕刻作品对于其作品细节和作品选材方面 都能给人以此类的潜意识认知, 并且在上千年的工艺 雕刻历史传承中, 工艺雕刻师们传承了中华民族独有 的文化鬼米力和厚重的文化积淀, 而这恰好也正是工艺 雕刻的负空间心理层面即潜意识应该着重注意的点; 即通过空间意识创新工艺雕刻同时不能忽视人们心 理层面意识的关注。

最后从工艺雕刻的意向层面出发; 工艺雕刻文化 属性是影响其负空间的关键内涵, 不同的时代有着属 于自己不同的文化属性, 所以工艺雕刻不仅要自我发 展还要通过当代的新思路、新文化让自己与时俱进从 而适应当代文化属性的步伐; 而对于文化属性的探 索, 一定离不开我国的主流意识形体, 要在一定程度 上摒弃其封建、腐朽的设计思路，而是在社会主义核 心价值观下去探索当代文化属性下的审美趋向。

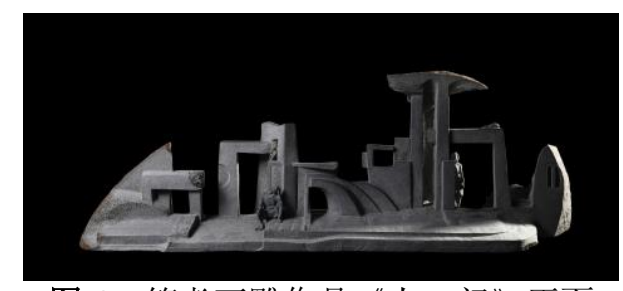

图 4 笔者石雕作品《人・门》正面

根据以上对其工艺雕刻在空间上的阐述, 笔者在 此基础上用常见的青石材料进行了工艺雕刻在空间 上的延展; 艺术来源于生活, 而工艺美术则是艺术门 类中最贴近我们生活的，我们的生活与工艺美术之间 
的关系更为密不可分; 笔者的作品《人・门》的来源 就是针对生活中随处可见但是又各不相同的门, 看似 简单的一扇门实则有其更深层的涵义; 首先, 门是对 于一个空间的分隔同时又是对于两个空间的连接, 他 就像一个岔路口一样只有两个选择即进或者是出, 恰 恰好就如同我们的生活道路一样, 在每每关键时刻留 给我们选择只有一二; 很多扇门组合在一起就像是我 们的人生遇到很多至关重要的选择一样。当时想到这 个选题时笔者正在自己人生道路上进行重大选择, 相 信对于大家都是一样的苦闷, 在门前犹豫对着门发 呆, 直到现在笔者还清晰的记着那扇门的模样; 其次 门与人之间有一种不可言喻的羁绊, 而这种羁绊的砝 码正是我们自己对过去的一种思念, 同时也是赋予门 有一定生命的源泉; 正是我们的存在, 门才有了特殊 的含义。

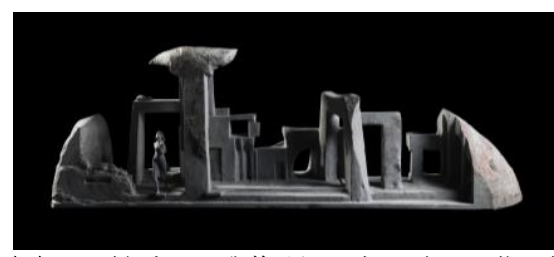

图 5 笔者石雕作品《人・门》背面

《人・门》这件作品在正空间上展现了一扇扇的 门与人之间的关系, 门是空间的链接, 它作为一个具 象的物质在与人一起发挥着抽象的互动, 一个门若有 了人在周围则会产生若干个负空间, 正如作品上的多 个奇形怪状的门一样与单独的两个人产生了抽象的 负空间, 而这样的负空间给予观者无限的想象, 而这 就是作品中空间性的展现; 其二对于作品的心理层 面, 笔者认为每个人面对很多门的时候都会去进行抉 择和思考, 而这种抉择和思考也恰恰让人们面对门时 有了定向思维, 而用第三者的态度去观看这种定向思 维也正是笔者在空间的心理层面的探索; 最后的意向 层面, 笔者在其中国传统思想文化基础和主流意识形 态层面上, 找到中国人潜在的文化内涵, 加以当代新 的思路、新文化让其作品紧跟我国当代文化趋势, 同 时也更加符合当代人们的艺术审美。

\section{6. 结论}

本文通过对空间意义和形成原因以及艺术形态 的阐述和对古今空间作品详细分析, 探讨了当代工艺 雕刻创新的新路径一空间意识。通过对现代的禅文化 工艺美术雕刻作品的阐述不难发现新时代的禅文化 体现在作品空间意识的把握, 大家加强了整体作品的 圆融性即空间性, 营造出不再单单具象的作品, 而具 有空间的工艺雕刻作品也正是映祄了本时代工业文 明下人们心理空间极度萎缩急需通过一定物质媒介 而抚慰心灵这样的大背景; 对其空间意识对当代工艺 美术雕刻作品具有一定积极的作用, 同时也一定程度 上对新工艺创作思路发掘新的思维可能性。

\section{REFERENCES}

[1] Hu b.(1999) Contemporary sculpture art: towards a new space.Hunan Fine Arts Publishing House, Changsha

[2] Shu xc.(2019) Negative space form and origin of ancient sculpture art.Art Education Research, 15.42-43

[3] Yuan w, Fuan jl.(2019)An analysis of the negative space of traditional Chinese aesthetics from the perspective of virtual reality.Art Science and Technology,22.20-21

[4] $\mathrm{Wu}$ w.(2020)Research on positive and negative space in sculpture modeling.artwork, 10.70-71

[5] Shu xc.(2019) Negative space sculpture and urban place design. China Architecture \& Building Press, The Beijing.

[6] Wu sx.(2008) Sculpture Art. People's Fine Arts Publishing House,Beijing 